\title{
ESTUDO RETROSPECTIVO DA PREVALENCIA DE CARDIOPATIAS EM CÃES ATENDIDOS ENTRE 2015-2019 PELO LABORATÓRIO DE CARDIOLOGIA COMPARADA DO HOSPITAL VETERINÁRIO DA UFPR
}

(Retrospective study of prevalence of heart disease in dogs assisted between 2015 - 2019 by the Compared Cardiology Laboratory of the UFPR Veterinary Hospital)

Fabiana Rodrigues de Freitas, Letícia Orthey Cidral, Tainah Godzinski Pereira, Caroline Pereira Ribeiro, Danielle Shiguihara, Bruna Natali da Costa, Simone Tostes de Oliveira Stedile, Marlos Gonçalves Sousa

Universidade Federal do Paraná, Curitiba, Brasil.

*Correspondência: fabidefreitasr@gmail.com

RESUMO: Doenças cardíacas, congênitas ou adquiridas, representam grande parte da casuística do atendimento de cães. Estima-se que aproximadamente $10 \%$ dos atendimentos iniciais em cães ocorrem devido a doença cardíaca, e a doença mixomatosa valvar mitral (DMVM) corresponde à maioria das cardiopatias nessa espécie (Keene et al., 2019). A ecocardiografia é um exame complementar não invasivo, importante para o diagnóstico das cardiopatias. Assim, o objetivo deste estudo foi analisar a prevalência de cães cardiopatas diagnosticados por meio da ecocardiografia, atendidos pelo Laboratório de Cardiologia Comparada do Hospital Veterinário da UFPR, durante o período de 2015 a 2019. Foram descartados da amostra os pacientes com diagnósticos incompletos, e apenas o exame mais recente do mesmo animal foi considerado. Sendo assim, foram analisados 2703 cães, dos quais 1668 (54,4\%) possuíam pelo menos uma cardiopatia, 737/1668 (44,2\%) apresentaram duas doenças concomitantes, e 24/1668 (1,4\%) apresentaram três ou mais doenças conjuntas. Dentre os cardiopatas, 953/1668 eram fêmeas $(57,1 \%)$ e $715 / 1668$ eram machos (42,9\%). Cães sem raça definida foram os mais acometidos 552/1668 (33,1\%), seguidos das raças Poodle 235/1668 (14,1\%), Lhasa Apso 123/1668 (7,4\%), Pinscher 103/1668 (6,2\%), Dachshund 95/1668 (5,7\%) e demais raças 560/1668 (33,6\%). A maioria dos animais 1580/1668 (94,7\%) foi diagnosticada com cardiopatias adquiridas. Destes, 40/1580 $(2,5 \%)$ apresentavam idade entre dois e cinco anos, 548/1580 (34,7\%) entre seis e dez anos, $841 / 1580$ (52,3\%) entre 11 e 15 anos, 93/1580 (5,9\%) acima de 15 anos e 58/1580 (3,7\%) não tiveram suas idades informadas. Os outros $88 / 1668$ (5,3\%) cães apresentaram doenças congênitas, destes $21 / 88(23,9 \%)$ apresentavam menos de um ano de idade, $11 / 88(12,5 \%)$ entre dois e cinco anos, 32/88 (36,4\%) entre seis e dez anos, 21/88 (23,9\%) entre 11 e 15 anos, 1/88 $(1,1 \%)$ acima de 15 anos e 2/88 (2,3\%) não tiveram as idades informadas. A DMVM foi a cardiopatia mais diagnosticada, observada em 1560/1668 (93,5\%) cães. A segunda maior afecção foi a degeneração mixomatosa da valva tricúspide (DMVT), observada em 756/1668 (45,3\%) cães. O terceiro diagnóstico mais comum foi a cardiomiopatia dilatada acometendo 39/1668 (2,3\%) animais, seguido por neoplasias cardíacas observadas em 20/1668 (1,2\%) cães. As demais doenças tiveram menor prevalencia, afetando dez indivíduos ou menos, sendo elas: estenose aórtica, comunicação interatrial, estenose pulmonar, displasia de mitral, displasia de tricúspide, endocardite, cor pulmonale, comunicação interventricular, persistencia do ducto arterioso, estenose subaórtica, estenose mitral, persistencia da veia cava cranial esquerda, hérnia diafragmática peritoneopericárdica, dirofilariose, degeneração aórtica, coronária anômala, cardiomiopatia arritmogenica, aneurisma, tromboembolismo pulmonar, trombo em átrio direito, tetralogia de Fallot, miocardite, cor triatriatum sinister e calcificação aórtica, respectivamente. Dentre os pacientes que apresentavam mais de uma doença concomitante, a DMVM juntamente com a DMVT, acometeram 706/1668 (42,3\%) animais. O estudo de prevalência das cardiopatias auxilia na elaboração de diagnósticos diferenciais e, portanto, na escolha do plano terapeutico.

Palavras-chave: casuística; degeneração mixomatosa da valva mitral; degeneração mixomatosa da valva tricúspide; diagnóstico; ecocardiografia

Referencia: KEENE, B. W. et al. ACVIM consensus guidelines for the diagnosis and treatment of myxomatous mitral valve disease in dogs. Journal of veterinary internal medicine, v. 33, n. 3, p. 1127-1140, 2019. 